\title{
Successful Medical Treatment of Pulmonary Mucormycosis in Diabetic Patients
}

\author{
Feki $\mathbf{W}^{1 *}$, Sellami $\mathbf{S}^{1}$, Charfi $\mathbf{S}^{2}$, Ketata $\mathbf{W}^{1}$, Msaad $\mathbf{S}^{1}$, Bahloul $\mathbf{N}^{1}$, Ayadi $\mathbf{H}^{1}$ and Kammoun $\mathbf{S}^{1}$ \\ ${ }^{1}$ Pneumology Department, Hédi Chaker Hospital, Sfax University, Tunisia \\ ${ }^{2}$ Department of pathology, Habib Bourguiba Hospital, Sfax University, Tunisia
}

\begin{abstract}
Pulmonary mucormycosis is a relatively uncommon infection that is mostly observed among diabetic patients. We present here three cases of pulmonary mucormycosis as the cause of non-resolving pneumonia in diabetic patients. The goals of our review are to better characterize the population at risk, presenting symptoms, radiological appearance, diagnostic methods and to demonstrate the efficacy of medical therapy.
\end{abstract}

Keywords: Mucormycosis; Diabetes; Amphotericin B

\section{Introduction}

Pulmonary mucormycoses is a relatively uncommon infection that occurs mostly in immunocompromised persons. It is the second most common form of mucormycosis, accounting for more than $30 \%$ of infections. This form has a high mortality (40\%-76\%), and carries substantial morbidity in some cases, due to a rapid local progression and prominent angioinvasion [1]. The main route of human infections is inhalation [2]. Fungal spores are dispersed into the air from decaying material. The pulmonary form is characterized as a fungal infection of the pulmonary parenchyma and larger bronchi typified by extensive vascular thrombosis and tissue necrosis

We present here three cases of pulmonary mucormycosis as the cause of non-resolving pneumonia in diabetic patients. The goal of our review is to better characterize the population at risk, presenting symptoms, radiological appearance, diagnostic methods, therapy, and outcome.

\section{Case Reports}

\section{Case 1}

A 42-year-old white man, practicing the profession of farmer, with a medical history of type 1 diabets mellitus, was admitted to hospital with a 2-week history of dry cough without neither fever nor extrathoracique manifestations. Physical examination revealed thachypnoea with respiratory rate of 24 per min. His sputum was mucopurulent and no acid fast bacilli were seen. A chest-X-ray (CXR) (Figure 1a) showed right basal cavitary lesion measuring $3 \times 3 \mathrm{~cm}$. Laboratory studies revealed hyperglycemia of $18 \mathrm{mmol}$ and $\mathrm{HbAlC}$ of $11 \%$. The C-reactive protein (CRP) was also highly elevated (268 $\mathrm{mg} / \mathrm{l}$ ). A blood culture gave no growth after 48 hours incubation. The antibiotic amoxicilline/Ac clavulanique was administred daily for 10 days. On short term follow up there was no clinical nor radiological improvement. Thoracic computed tomographic (CT) (Figure 1b) showed many cavitory lesions involving all lobes. Flexible fiberoptic bronchoscopy performed on day 10 of his admission revealed a whitish coating on the lower right lobe with tasks burning candles. The mycological examination of bronchial fluid showed the presence of large non septate fungal filaments branched at right angles whose culture was not contributory (Figure 1c). Bronchial biopsies have found exudative necrosis rich nuclear debris, including a filamentous fungus aspect in Chinese alphabet which has been identified by special stains PAS and GROCOTT. Thus the diagnosis of pulmonary mucormycosis was retained and treatment with amphotericin B was started at a dose of $0.1 \mathrm{mg} / \mathrm{kg}$ doses with progression to $1 \mathrm{mg} / \mathrm{kg}$. The outcome was

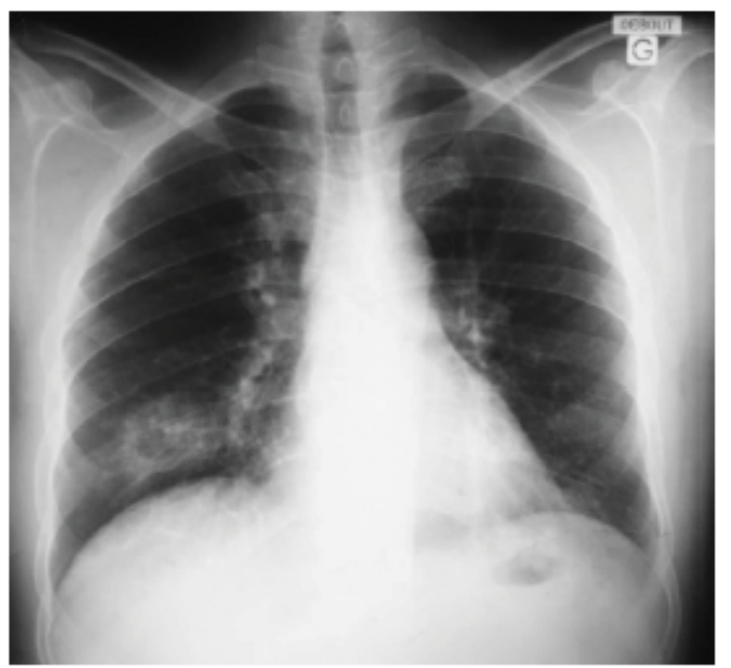

Figure 1(a): Right basal cavitary lesion measuring $3 \times 3 \mathrm{~cm}$.

favorable after 15 days of treatment with clear regression of clinical signs and progressive cleaning radiological images after 6 weeks.

\section{Case 2}

A 73-year-old male with uncontrolled type II diabetes mellitus of 20 years duration was admitted to hospital. He was a tobacco smoker (5 pack-years), presented with an 8-week history of low abundance hemoptysis and $10 \mathrm{~kg}$ weight loss. He denied fever, chills or night sweats. No close contact with persons with tuberculosis was reported. Physical examination revealed normal blood pressure, heart rate and breathing frequency. Oxygen saturation was $99 \%$ on room air. Chest examination revealed normal breath sound. The admission chest radiograph was initially interpreted as showing a bilateral consolidation measuring 6

${ }^{*}$ Corresponding author: Feki W, Pneumology Department, Hedi Chaker Hospital, Sfax University, Tunisia, Tel: 0021697051789; E-mail: Fki_walid@yahoo.fr

Received January 11, 2018; Accepted January 29, 2018; Published February 05, 2018

Citation: Feki W, Sellami S, Charfi S, Ketata W, Msaad S, et al. (2018) Successful Medical Treatment of Pulmonary Mucormycosis in Diabetic Patients. J Pulm Respir Med 8: 444. doi: 10.4172/2161-105X.1000444

Copyright: (c) 2018 Feki W, et al. This is an open-access article distributed under the terms of the Creative Commons Attribution License, which permits unrestricted use, distribution, and reproduction in any medium, provided the original author and source are credited. 
Citation: Feki W, Sellami S, Charfi S, Ketata W, Msaad S, et al. (2018) Successful Medical Treatment of Pulmonary Mucormycosis in Diabetic Patients. J Pulm Respir Med 8: 444. doi: 10.4172/2161-105X.1000444

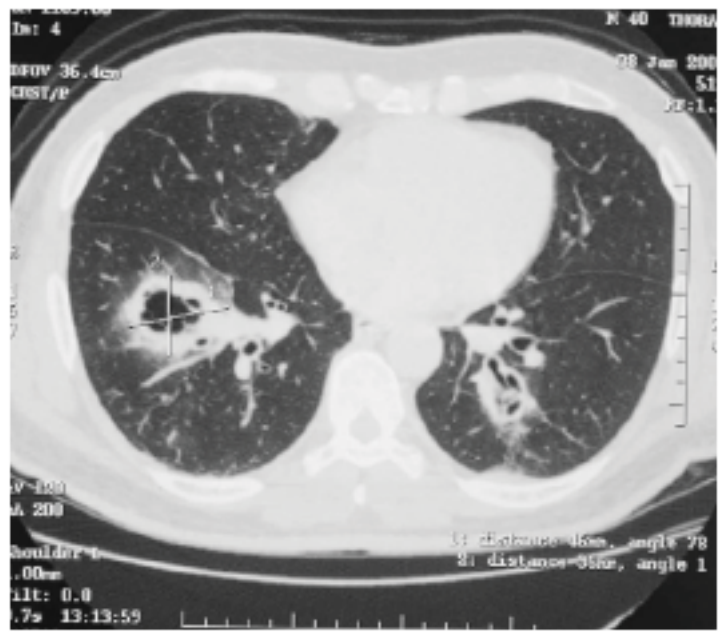

Figure 1(b): Many cavitory lesions involving all lobes.

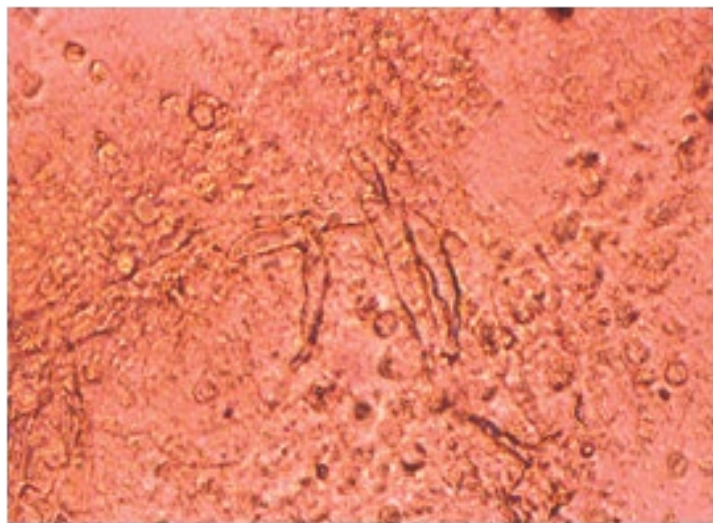

Figure 1(c): Large mycotic filaments without septa branched out at right angle on direct examination.

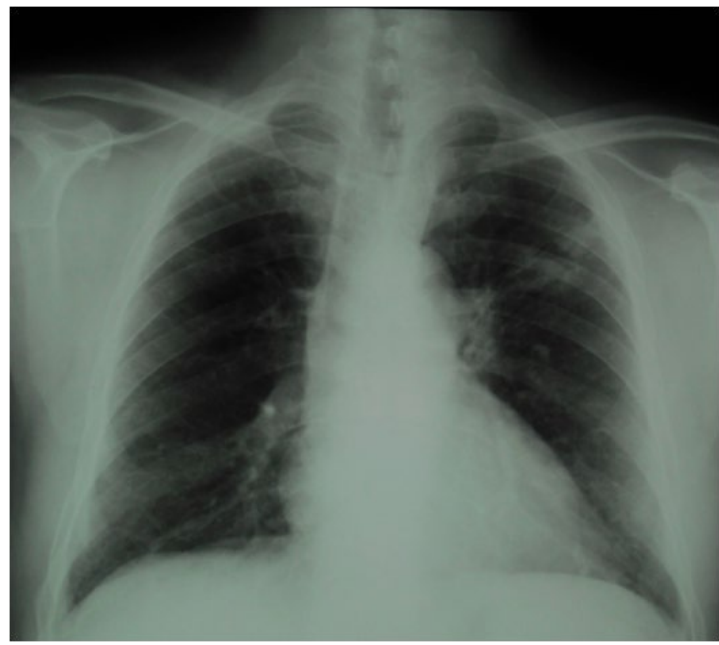

Figure 2(a): Left upper lobe heterogeneous opacity.

$\mathrm{cm}$ with many cavitory lesions and thickened pleura opposite (Figure 2a). Investigations revealed haemoglobin of $11.3 \mathrm{gm} \%$, total leucocytes count of 9,300 cells/cumm, an elevated erythrocyte sedimentation rate (65 mm/hr). He had severe hyperglycemia (blood glucose $410 \mathrm{mg} / \mathrm{dl}$ ), acidosis $\left(\mathrm{PaO}_{2} 100 \mathrm{mmHg}, \mathrm{HCO}_{3}-14 \mathrm{mmol} / \mathrm{L}\right.$ and $\mathrm{pH}$ of 7.22$)$ and ketonuria $(+++)$. The hydatid, aspergillus and HIV serologies were negative. There was no evidence of acid-fast bacilli in the sputum. Contrast enhanced Computed Tomography chest showed a left upper lobe mass round, $5 \mathrm{~cm}$ in diameter, excavated center and regular wall enhanced after injection of contrast with epsilateral hilar and mediastina lymph node enlargement (Figure $2 b$ ). This mass comes into contact with the great fissure and adjacent thickened parietal pleura. We suspected the mass to be malignant and went for CT-guided true cut biopsy. Histology consistant with mucor infection. Computed tomography scan of the head and sinuses was normal. The patient was treated for diabetic ketoacidosis with intravenous saline, insulin infusion and appropriate management for hypokalemia. Liposomal amphotericin B in the dose of $0.75 \mathrm{mg} / \mathrm{kg}$ every day was started. $\mathrm{He}$ completed 6 weeks of amphotericin B, but developed renal failure, possibly from amphotericin $B$, and was reduced than stopped. When last evaluated, 6 months following the initial presentation, the patient was asymptomatic, with a stable chest CT (Figure 2c).

\section{Case 3}

An 82-year-old man, with a 20-year history of uncontrolled diabetes and a heart rhythm disorder, presented to the emergency

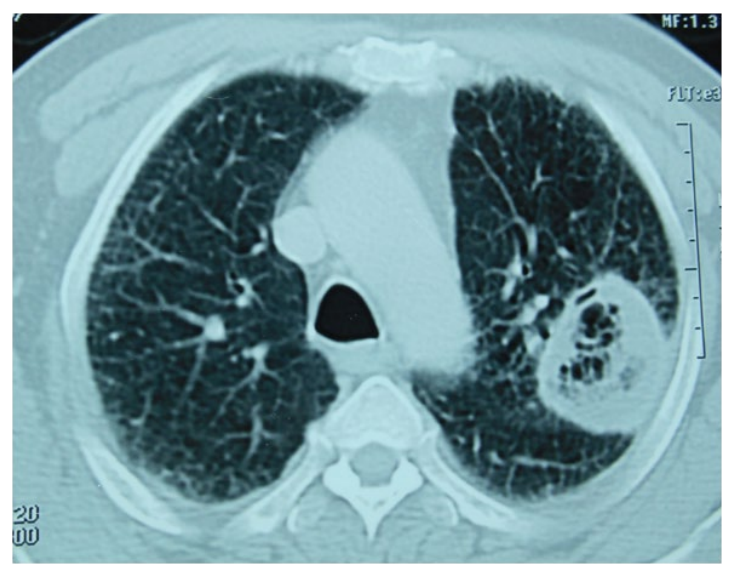

Figure 2(b): A left upper lobe mass round, $5 \mathrm{~cm}$ in diameter, excavated center and regular wall enhanced after injection of contrast with epsilateral hilar and mediastina lymph node enlargement.

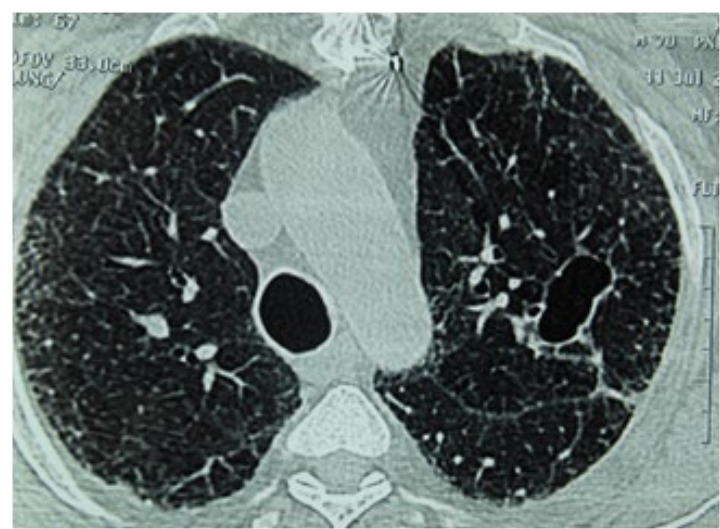

Figure 2(c): Stable computed tomographic after treatment. 
Citation: Feki W, Sellami S, Charfi S, Ketata W, Msaad S, et al. (2018) Successful Medical Treatment of Pulmonary Mucormycosis in Diabetic Patients. J Pulm Respir Med 8: 444. doi: 10.4172/2161-105X.1000444

department with a 5-months history of middle abundance hemoptysis. He did not have substantial smoking history. On examination, he had a pulse rate of $110 / \mathrm{min}$, blood pressure $133 / 75 \mathrm{mmHg}$, and respirations 24/min. Examination of the respiratory system revealed bilateral crackles. The remainder of the patient's physical examination was unremarkable. Routine blood and biochemistry showed hemoglobin was $8.2 \mathrm{gm} / \mathrm{dL}$, total white blood cell count was 7600 per $\mathrm{mm}^{3}$ with neutrophil comprising $77 \%$, fasting sugar was $510 \mathrm{mg} / \mathrm{dL}$, serum levels creatinine was $1.1 \mathrm{mg} / \mathrm{dL}$. CXR showed bilateral consolidations with many cavitory lesions and right pleural effusion (Figure 3a). Aspergillus antigen testing of serum specimens was negative. Sputum examination revealed no acid-fast bacilli on Ziehl-Neelson staining. CT of the chest revealed bilateral cavitating lesions with a reversed halo sign (Figure $3 b)$. Fibreoptic bronchoscopy revealed congested bronchial mucosa. Fungal culture remained negative, but histological examination by an experienced mycologist revealed broad, non-septate fungal hyphae with branches occurring at right angles. The morphology of hyphae was suggestive for moulds belonging to the order mucorales and causing mucormycosis (Figures $3 \mathrm{c}$ and $3 \mathrm{~d}$ ). Computed tomography scan of the head and sinuses was normal. Diabetic control was achieved with insulin. Empirical treatment with conventional amphotericin B (cAmB) was started at dose of $0.8 \mathrm{mg} / \mathrm{kg} /$ day. On day 30 after beginning

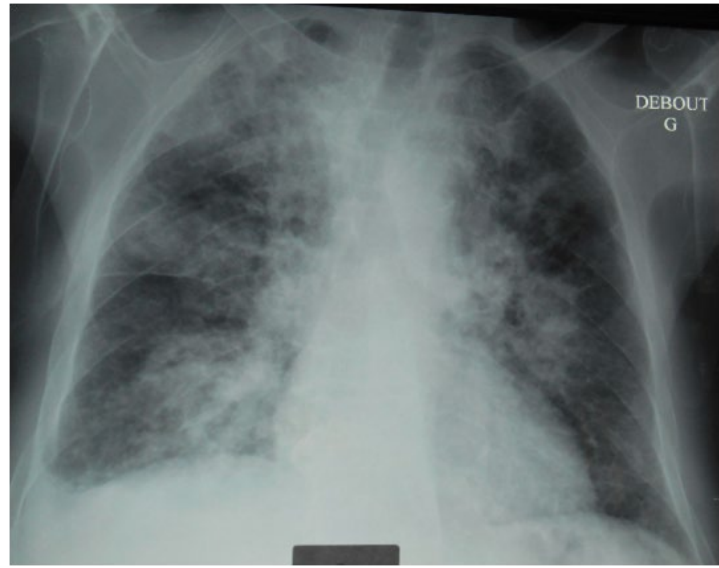

Figure 3(a): Bilateral consolidation with many cavitory lesions and right pleural effusion.

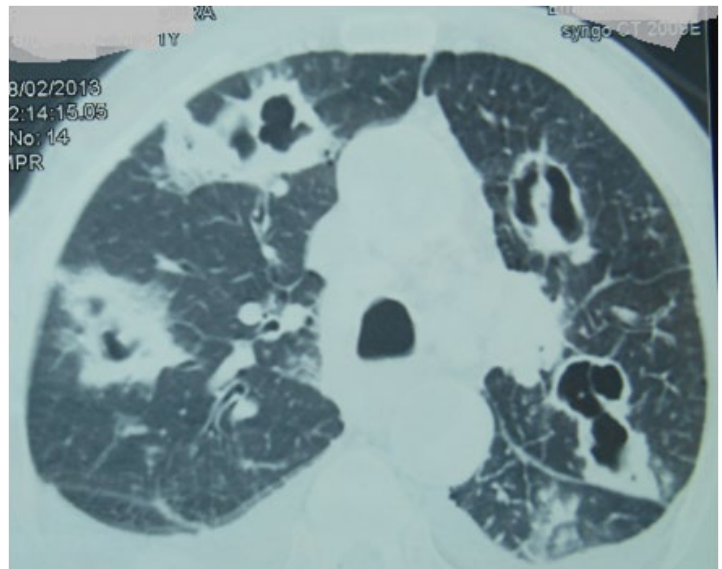

Figure 3(b): Bilateral cavitating lesions with a reversed halo sign associated with bilateral pleural effusion.

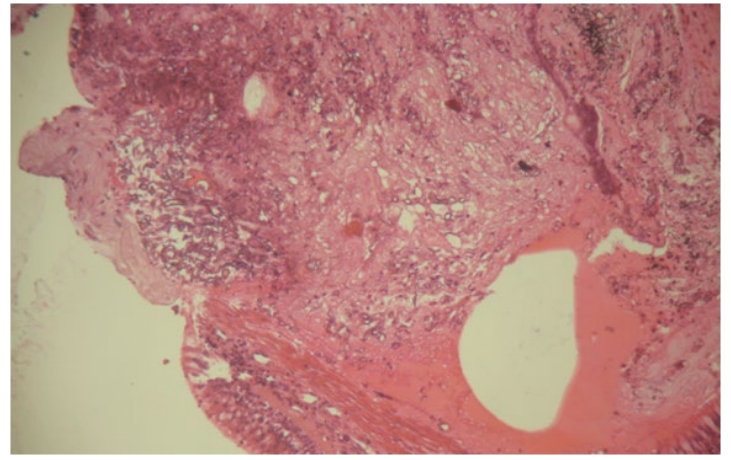

Figure 3(c): Fungal infiltration of the branchial mucosa (H\&E 40X).

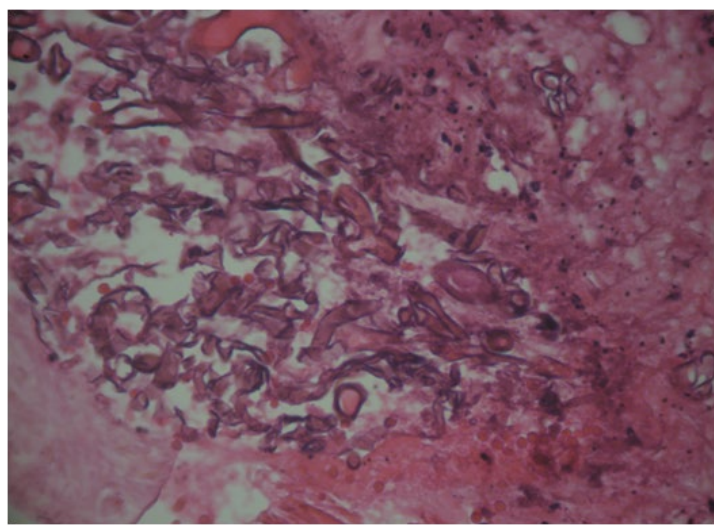

Figure 3(d): fungal hyphaes are thick, irregular and non-septated with rightangle branching (H\&E 400X).

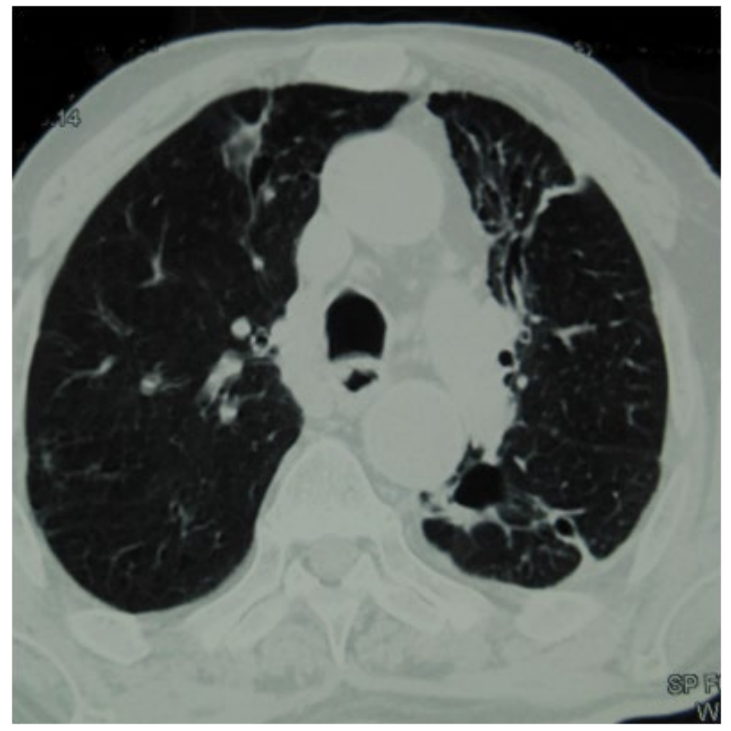

Figure 3(e): Marked resolution of the parenchymal lesions.

fungal therapy, a clinical and radiological improvement was observed. However, the patient developed acute renal failure (creatinine 170 $\mu \mathrm{mol} / \mathrm{l})$. Amphotericin B dose was reduced to $0.5 \mathrm{mg} / \mathrm{kg} /$ day and the renal function remaind stable (creatinine $130 \mu \mathrm{mol} / \mathrm{l}$ ). 
Subsequently, a CT of the chest repeated after 6 weeks showed marked resolution of the parenchymal lesions. The fungal treatment was stopped after 4 months. More than 1 year after treatment, the follow-up period was uneventful, the patient remains stable without any clinical or radiological evidence of recurrence (Figure 3e). He continues to be seen regularly in our department.

\section{Discussion}

Mucormycosis is a group of devastating opportunistic infections caused by moulds from the order Mucorales in the Zygomycetes class. The most common presentation is rhino-orbital-cerebral involvement followed by pulmonary infection. Pulmonary mucormycosis is defined as a disease localized in the lungs or mediastinum [3]. The first case of pulmonary mucormycosis was reported in 1876 by Fur Bringer [4]. Pulmonary involvement can occur as a result of inhalation of fungal spores or from hematogenous or lymphatic spread from a distal focus, rhinocerebral or cutaneous. It is a relatively uncommon infection occurring predominantly in immune-compromised hosts. Numerous predisposing factors have been suggested for mucormycosis. One of the most common is poorly controlled diabetes mellitus and ketoacidosis [5]. Type 1 , type 2 , and secondary diabetes mellitus are all reportedly risk factors for mucormycosis. Acidosis may promote fungal growth by altering the binding of iron to serum proteins. Mucormycosis may be the first manifestation in some patients with undiagnosed diabetes mellitus [6], but it is rarely observed in those with metabolically controlled diabetes

The clinical features of pulmonary mucormycosis are nonspecific. Its cannot be easily distinguished from those of other pulmonary infections. Patient can present with mild to severe symptoms. Usually, there was prolonged high-grade fever that is unresponsive to broadspectrum antibiotics. Nonproductive cough and hemoptysis were common, whereas, pleuritic chest pain, and dyspnea are less common [7]. Chest physical examination may show rales, evidence of pulmonary consolidation, or not uncommonly pleural friction rubs.

Radiographically, a variety of findings may be present. On chest imaging, pulmonary mucormycosis may present with infiltration, focal consolidation, lung masses, pleural effusions and even normal findings [8]. Nodules can be unilateral or bilateral, solitary or multiple. Cavitary lesions with the "air crescent sign" have been described, but are rare. High-resolution chest CT scan is the best method of determining the extent of pulmonary mucormycoses and may demonstrate evidence of infection before it is seen on the chest X-ray. Jamadar et al. [9] revived the CT appearance of pulmonary mucormycosis and found a prediction for upper lobes and cited caritation, air crescent sign, halo sign and rim enhancement as radiological evidence of necrosis in these patients. One of our three patients had this radiographic finding (halo sign).

Traditionally, an early diagnosis of pulmonary mucormycosis is difficult because of the rapid course of this often fatal illness, the variability of clinical presentation and the rarity of the infection. Therefore, the most widely used successful method of diagnosis is the visualization of the fungi upon microscopic examination, of diseased tissue collected during flexible fiberoptic bronchoscopy [3]. The typical bronchoscopic findings are bronchial stenosis or obstruction, erythematous mucosa, gelatinous or mucoid secretions, fungating or polypoid masses, and mucosal ulceration. Transthoracic needle aspiration as a diagnostic tool of mucormycosis was used only in a small minority of patients (10\%-20\%). Fungal culture of the specimens was found to be positive in half of the patients making it as a relatively insensitive tool. The histopathological findings reveal irregular broad non septate hyphae and spores with right angled, irregular branching and a few rosettes of needle shaped crystals stained with methanamine silver or periodic acid-Schiff stain [10].

Because of rapid local invasion and possibility of haematogenous dissemination with a fatal course in more than $50 \%$ of cases (3), an early and aggressive treatment is imperative. Several studies have shown that the combination of early surgical resection and antifungal therapy has a significant improvement in survival, when compared to antifungal therapy alone [11]. Liposomal amphotericin $B$ is the mainstay therapy, and should be promptly initiated once the diagnosis is confirmed. But there are concerns about the limited penetration of antifungals to the affected tissues, due to the substantial necrosis that accompanies this infection [12]. For this reason, surgical debridement has been advocated. Although wedge resection may suffice, lobectomy is the usual procedure. If the infection crosses tissue planes, pulmonary resection and debridement of adjacent structures is required [13].

If the patient shows rapid and continued response, medical therapy may be sufficient as observed in our case. The duration of therapy is individualized to the patient, but the near normalization of radiographic abnormalities, negativity of cultures, or resolution of the immunosuppressed state can be used as surrogates to stop therapy.

However, the total dose needed for cure remains not well defined. The appropriate time period between the initiation of medical therapy and surgery is still indeterminate, usually extended until regression of the invasion under antifungal therapy is obtained [14]. Controlling of the patient's underlying disease is essential in the experience with mucormycosis. A highly unusual outcome with spontaneous resolving pulmonary mucormycosis has been reported in one patient without treatment after correction of ketoacidosis [15]. When fatal, the most common causes of death include fungal sepsis, pulmonary dysfunction, and/or hemoptysis [7]. The only effective antifungal agents for treatment of mucormycosis are different formulations of amphotericin $\mathrm{B}$ and the newest triazole agent, posaconazole. The use of $\mathrm{AmB}$ is, however, hampered by the need of high doses and considerable toxicity of this agent with prolonged use. Low nephrotoxicity and uncommon infusion related adverse effects make LAmB a promising alternative in the treatment of mucormycosis

Survival seems to have increased. Substantially, more patients are now being diagnosed premortem, which may help explain the better outcome. In Baker's article [16], $12 \%$ of the patients survived. In 1977, Murray [17] noted in an editorial that only $9 \%$ with localized pulmonary involvement survived in the first 100 years since the disease was originally described.

Pulmonary mucormycosis is a rare but emerging fungal infection with a high mortality rate. Proper and immediate treatment can significantly improve the prognosis.

\section{References}

1. Smith JA, Kauffman CA (2012) Pulmonary fungal infections. Respirol 17: 913-926

2. Gonzalez EC, Couriel RD (1997) Disseminated zygomycosis in a neutropenic patient: Successful treatment with amphotericin b lipid complex and granulocyte colony-stimulating factor. Clin Infec Dis 24: 192-196.

3. Lee FY, Mossad SB, Adal KA (1999) Pulmonary mucormycosis: The last 30 years. Arch Int Med 159: 1301-1309.

4. Fürbringer $P$ (1876) Beobachtungen über lungenmycose beim menschen. Archiv für Pathologische Anatomie Physiologie für Klinische Medicin 66: 330-365.

5. Donohue JF (1983) Endobronchial mucormycosis. Chest 83: 585

6. Kontoyiannis DP (2007) Decrease in the number of reported cases of 
Citation: Feki W, Sellami S, Charfi S, Ketata W, Msaad S, et al. (2018) Successful Medical Treatment of Pulmonary Mucormycosis in Diabetic Patients. J Pulm Respir Med 8: 444. doi: 10.4172/2161-105X.1000444

zygomycosis among patients with diabetes mellitus : A hypothesis. Clinical infec Dis 44: 1089-1090.

7. Tedder M, Spratt JA, Anstadt MP, Hegde SS, Tedder SD, et al. (1994) Pulmonary mucormycosis: Results of medical and surgical therapy. Annals Thoracic Surg 57: 1044-1050.

8. Bartter T, Irwin RS, Nash G () Aneurysms of the pulmonary arteries. Chest 94 1065-1075.

9. Jamadar DA, Kazerooni EA, Daly BD, White CS, Gross BH (1995) Pulmonary zygomycosis: CT appearance. J Comput Assist Tomogr 19: 733-738.

10. Tazelaar HD, Baird AM, Mill M, Grimes MM, Schulman LL, et al. (1989) Bronchocentric mycosis occurring in transplant recipients. Chest 96: 92-95.

11. Saegeman V, Maertens J, Ectors N, Meersseman W, Lagrou K (2010) Epidemiology of mucormycosis: Review of 18 cases in a tertiary care hospital. Medical Mycol 48: 245-254.
12. Spellberg B, Ibrahim AS (2010) Recent advances in the treatment of mucormycosis. Current Infec Dis Rep 12: 423-429.

13. Fitzpatrick MC, Carter BW (2012) Pulmonary mucormycosis complicating cutaneous blastic plasmacytoid dendritic cell neoplasm. Proc (Bayl Univ Med Cent 25: 287-288.

14. Reid VJ, Solnik DL, Daskalakis T, Sheka KP (2004) Management of bronchovascular mucormycosis in a diabetic: a surgical success. Annals Thoracic Surg 78: 1449-1451.

15. Mendoza-Ayala R, Tapia R, Salathe M (1999) Spontaneously resolving pulmonary mucormycosis. Clin Inf Dis 29: 1335-1336.

16. Wingard JR, Kubilis P, Lee L, Yee G, White M, et al. (1999) Clinical significance of nephrotoxicity in patients treated with amphotericin $B$ for suspected or proven aspergillosis. Clin Inf Dis 29: 1402-1407.

17. Murray HW (1977) Pulmonary mucormycosis: One hundred years later. Chest 72: $1-2$ 